\title{
Directional plasmonic scattering from metal nanoparticles in thin-film environments
}

\author{
A. W. Powell, ${ }^{\text {a) }}$ N. Hjerrild, A. A. R. Watt, ${ }^{\text {b) }}$ H. E. Assender, ${ }^{\text {c) }}$ and J. M. Smith ${ }^{\text {d) }}$ \\ Department of Materials, University of Oxford, Parks Road, Oxford OXI 3PH, United Kingdom
}

(Received 17 January 2014; accepted 11 February 2014; published online 25 February 2014)

\begin{abstract}
The effect of a low refractive index film on plasmonic scattering from individual silver nanoparticles and nanowires is observed experimentally in Fourier space. The addition of the film over particles on a glass substrate was found to have a strong effect on the scattering pattern, due to interference between directly scattered light and light reflected from the interfaces, which depends strongly on the film thickness. Nanowires are shown to produce a much more directional scattering pattern than spherical particles, and the addition of the film above the nanowires leads to further directionality enhancements with light scattering at well-defined angles into the glass substrate. The use of the dielectric environment and particle shape to tune the directional scattering is demonstrated. These results are of interest for applications such as optical nanoantennae and plasmonically enhanced solar cells. (C) 2014 AIP Publishing LLC.
\end{abstract}

[http://dx.doi.org/10.1063/1.4866669]

The excitation of localised surface plasmons (LSP's) in noble-metal nanostructures offers a promising means to control light-matter interactions on the sub-wavelength scale and is currently the subject of intense research for a variety of applications including nanoantennae, ${ }^{1-3}$ single molecule detection, ${ }^{4,5}$ thin-film solar cells, ${ }^{6-8}$ and light-emitting diodes. ${ }^{9}$ LSP's are attractive due to their strong, resonant interaction with incident light, and the sensitivity of these resonances to particle size, shape, and surroundings. By altering these conditions, it is possible to manipulate the spectrum, near-field behaviour and directional scattering of plasmonic particles.

There have been several studies investigating the spectral properties of plasmonic nanoparticles (NP's) in various media, ${ }^{10-13}$ but only a few investigating their spatial scattering behaviour. Significantly, all the previous experimental papers investigating scattering directionality have only considered plasmonic particles at a substrate-air interface ${ }^{14-16}$ and the potential benefits of utilising a more structured dielectric environment have not been addressed. Directional emission in the form of leakage radiation from surface plasmon-polaritons has also been investigated, ${ }^{17,18}$ which is due to a very different mechanisms than the LSP scattering discussed here and suffers from strong absorption of the plasmon wave in the metal.

In this Letter, we demonstrate experimentally how the directional scattering behaviour of individual silver nanoparticles and nanowires (NW's) can be manipulated by controlling the thin-film environment of the scatterer. Altering the film thickness provides a simple means of fine-tuning scattering patterns. It is found that greater film thicknesses result in larger scattering angles and that at certain values the directionality is maximised, especially in the case of nanowires.

\footnotetext{
${ }^{\text {a)} E l e c t r o n i c ~ m a i l: ~ a l e x a n d e r . p o w e l l @ m a t e r i a l s . o x . a c . u k ~}$

${ }^{b}$ URL: http://www-solar.materials.ox.ac.uk/

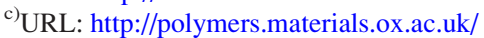

${ }^{\text {d) URL: http://www-png.materials.ox.ac.uk }}$
}

We compare experimental data with finite-difference timedomain (FDTD) simulations to verify our findings.

The plasmonic dipole mode generally has the largest optical cross-section and suffers smaller losses due to plasmonic absorption compared with higher-order $\operatorname{modes}^{10}$ and is thus the preferred excitation for most applications. Placement of a dipolar emitter or scatterer at the interface of two extended media, e.g., air and glass, will result in a different scattering patterns from free space due to interference between directly emitted light and light reflected from the interface. ${ }^{19}$ The addition of any films or other media around the NP will alter the interference and strongly affect the emission/scattering pattern as well as the spectral properties. This can lead to longer optical path lengths in

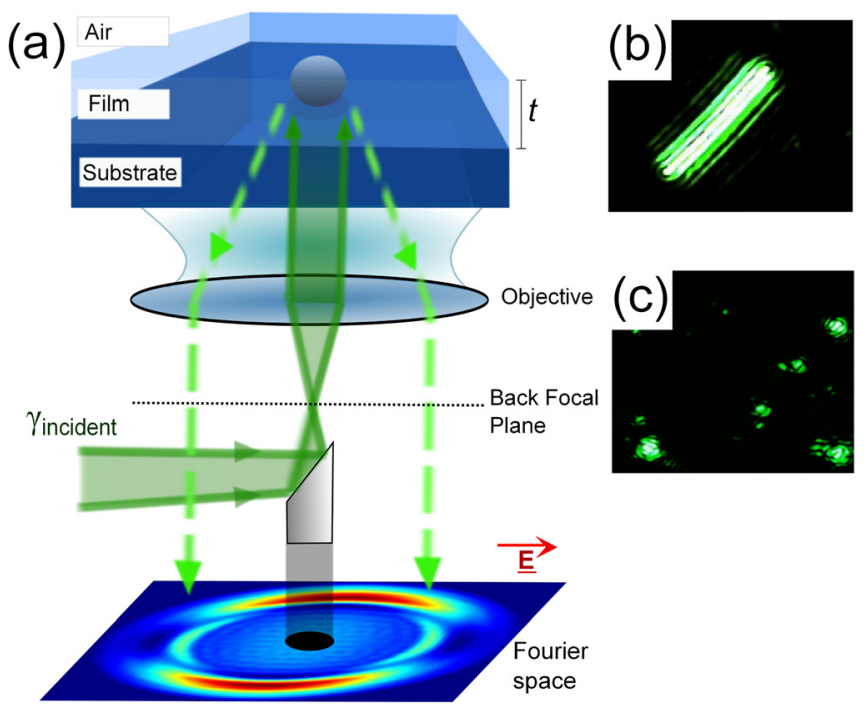

FIG. 1. (a) Diagram illustrating the key principles behind the dark-field Fourier microscope. Light is reflected from the rod mirror onto the sample, is scattered by particles back through the objective and then mapped onto the Fourier plane as a function of angle, also shown. Low angle light is blocked by the mirror-resulting in a dark patch at the centre of the image. Real space, dark field images of (b) a silver nanowire, and (c) silver nanoparticles taken using the microscope are also shown. 
photodetectors, higher collection efficiency for fluorescence microscopy, and greater control over the directional emission for nanoantennae. ${ }^{11,20,21}$

Samples were prepared using spherical silver nanoparticles, procured from Sigma Aldrich and Ag nanowires produced in house using a polyol reduction process ${ }^{22}$ were spincoated onto glass coverslips with a refractive index of 1.526 . The particles were imaged in a JEOL $840 \mathrm{~F}$ scanning electron microscope (Supplementary material, ${ }^{23}$ Fig. S1) and found to have an average diameter of $107 \pm 2 \mathrm{~nm}$. The nanowires had an average diameter of $115 \pm 3 \mathrm{~nm}$ and varied in length between 3 and $20 \mu \mathrm{m}$. Thin films were produced using the low index polymer Poly[4,5-difluoro-2,2-bis(trifluoromethyl)-1,3-dioxole-co-tetrafluoroethylene] (PTFE AF), from Dupont (which has a refractive index of 1.31) dissolved in the Fluorinert ${ }^{\mathrm{TM}}$ liquid FC-40 from $3 \mathrm{M}$. A spin coater was used to overcoat the particles with films of controllable thickness, $t$, which were then measured using a Pacific Nanotechnology Nano-R atomic force microscope in tapping mode.

Figure 1(a) shows the sample geometry and illustrates the dark-field, Fourier microscope used to image the scattering patterns (For a full diagram showing the complete optical setup, see supplementary material, ${ }^{23}$ Fig. S2). Laser excitation at $\lambda=532 \mathrm{~nm}$ is focussed into the back focal plane of an Olympus 100x, 1.4 numerical aperture (NA), oil immersion objective via a $1 \mathrm{~mm}$ rod mirror so it arrives collimated at the focal plane. LSP's are excited in particles on the back surface of a cover slip, where because of the higher refractive index of glass, the majority of the scattered light is directed back towards the objective, this is then mapped onto the Fourier plane as a function of angle. ${ }^{19}$ All light directly scattered from the interfaces is blocked by the rod mirror, which appears in the Fourier images as a dark spot around the centre. The microscope is aligned such that it is possible to visualise the Fourier and image planes simultaneously. Figs. 1(b) and 1(c) show optical dark-field images of an NW and several NP's taken using the microscope. A variable aperture is used to select single particles for Fourier space measurements. The far-field scattering patterns for each thickness were simulated using the Lumerical FDTD Solutions software.

Figure 2 shows the simulated (a)-(d) and measured (e)-(h) Fourier patterns of NP's at a bare interface and overcoated with $89 \pm 7,185 \pm 5$, and $247 \pm 4 \mathrm{~nm}$ films. Simulations were conducted using the average measured film thicknesses and particle sizes. The normalised line traces across the vertical axes of experimental and simulated plots are also shown in (i)-(l). The scattering pattern is shown to be defined by the thickness of the film containing the particle. As $t$ increases from (a)-(d), the lobe peak moves outwards from $41^{\circ}$ to $54^{\circ}$ from the optical axis and the emission at low angles reduces from $50 \%$ of the peak value in Fig. 2(i) to $17 \%$ in Fig. 2(k), before increasing again and beginning to form a secondary pair of lobes, which have just started to emerge in Fig. 2(1). The experimental results show strong agreement with simulations, once the mirror patchstop at the centre and the NA at the edges are taken into account.

This behaviour is in agreement with the theory of dipolar emitters in multi-layered structures as defined by Lukosz $^{24}$ and is due to the interference between directly scattered light and light then reflected from the film/substrate

\section{Bare Glass}

(a)

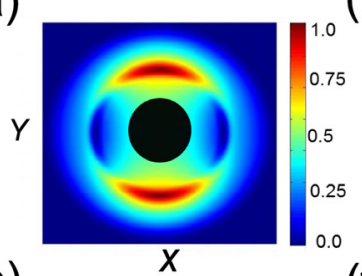

(e)
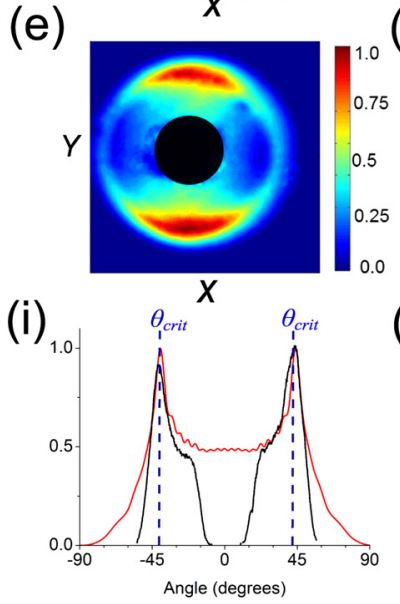

$89 \mathrm{~nm}$ film

(b)

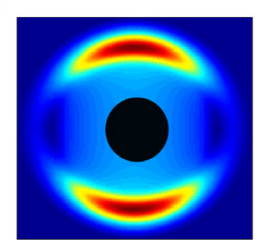

(f)

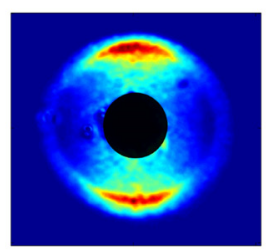

(j)

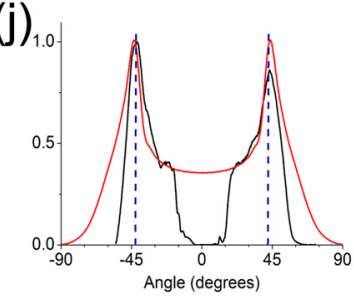

$185 \mathrm{~nm}$ film

(c)

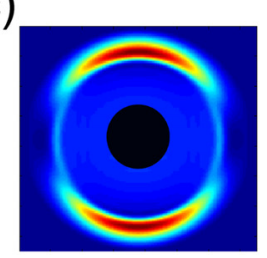

(g)

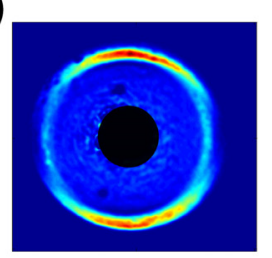

$(k)_{1.0}$

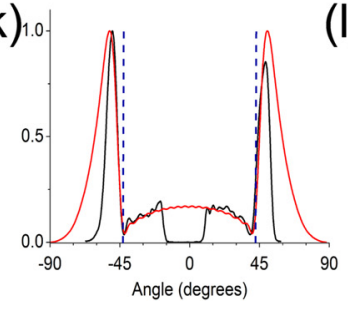

$247 \mathrm{~nm}$ film

(d)

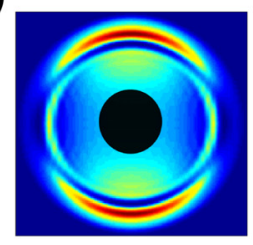

(h)

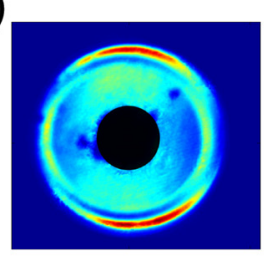

(I)

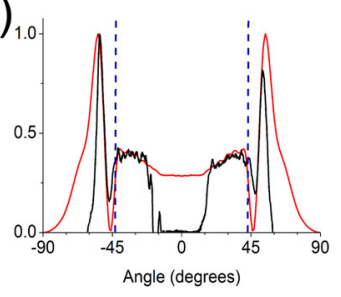

FIG. 2. FDTD simulated (a)-(d) and experimental (e)-(h) Fourier space images of scattering patterns from $107 \mathrm{~nm}$ Ag nanospheres at an air-glass interface ((a), (e), and (i)) and overcoated with $89 \pm 7 \mathrm{~nm}((\mathrm{~b})$, (f), and (j)), $185 \pm 5 \mathrm{~nm}$ ((c), (g), and (k)) and $247 \pm 4 \mathrm{~nm}((\mathrm{~d})$, (h), and (1)) of PTFE AF. Plots (i)-(j) show line traces across the vertical axis of all plots to better compare theory (red lines) and experiment (black lines). The air-glass critical angle $\left(\theta_{\text {crit }}\right)$ is displayed as a dashed blue line. 
and film/air interfaces. When the refractive indices fulfil $n_{\text {air }}<n_{\text {film }}<n_{\text {substrate }}$ it can be demonstrated that this interference is determined chiefly by reflection from the film/air interface $^{20}$ in such a way that as the film thickness increases, the interference maxima shift to larger angles and new maxima begin to form close to the center, until $t$ becomes large compared with the wavelength and we return to the pattern of a particle at a single interface. Thus, the majority of the light will be scattered into one or more pairs of well-defined lobes, and the concentration of light within a given angular spread will be dependent on $t$. Previous studies ${ }^{11}$ have shown that the same effect can be achieved by altering the refractive index of the film, as this produces an equivalent change to the optical path length.

For many applications, the ability to scatter light to the largest possible angles, thus maximising path length in the substrate through careful choice of layer materials and geometries is extremely useful. For antennae however, it is important to be able to scatter or emit light into as well defined a region as possible. For this purpose, nanowires offer an attractive alternative without the need to create more complex shapes. ${ }^{25}$

It has been demonstrated that a nanowire can be modelled as a set of discrete point dipoles arranged in a line across the length of the wire. ${ }^{14}$ Due to the phase coherence of scattered light from different points along the wire, the dipole radiation pattern is multiplied by a form factor, obtained by integrating over the wire length. ${ }^{16}$ This results in the predicted scattering pattern being that of a dipole, relative to the polarisation of the exciting radiation, multiplied by a $\operatorname{sinc}^{2}$ function arising from the wire geometry (see supplementary material ${ }^{23}$ ). The larger the wire aspect ratio the more the light is confined to the axis perpendicular to the wire.

Figure 3 shows the experimentally obtained scattering patterns from an Ag nanowire on a glass substrate, under
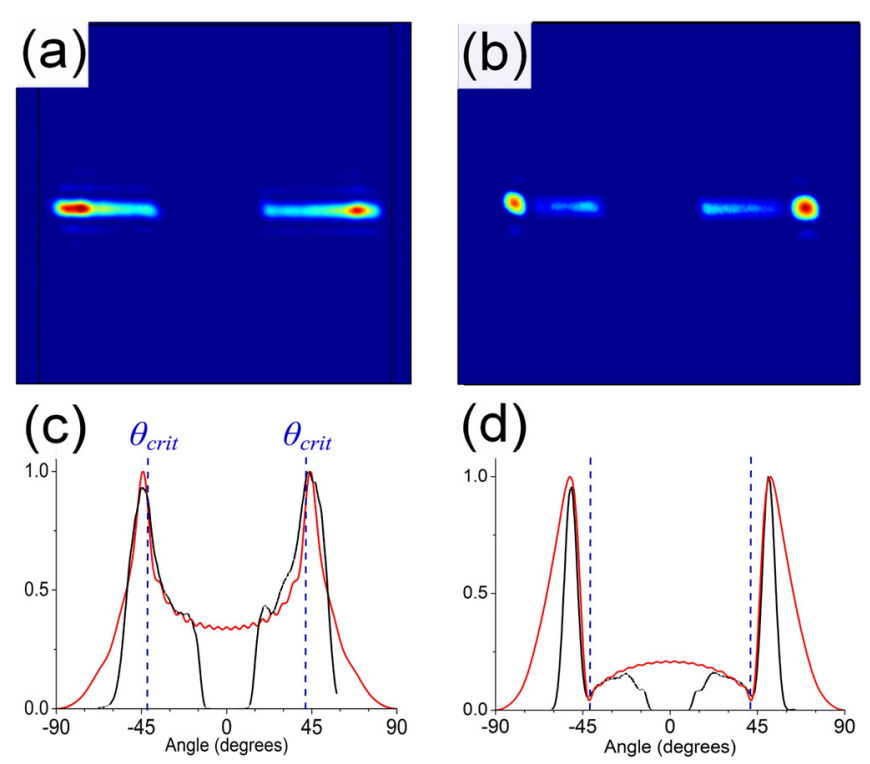

FIG. 3. Experimental Fourier space images and line traces of scattering from $115 \mathrm{~nm}$ diameter NW's on the surface of a glass coverslip with incident radiation polarised parallel to the wire axis. (a) and (c) show results for a wire on bare glass, (b) and (d) for a NW overcoated with $185 \pm 5 \mathrm{~nm}$ PFTE AF. The line trace plots also show results from FDTD simulations. illumination polarised parallel to the wire axis. Light polarised perpendicular to the wire produces a weaker scattering effect and shows less marked variation with $t$ (see Fig. S3, Ref. 23). For NW's on a bare glass substrate, the lobe peak is 2.9 times the value at $0^{\circ}$ and $37.7 \%$ of the light scattered into the substrate being caught within $10^{\circ}$ of the lobe peaks, which is a significant increase compared to $2.9 \%$ for a NP at a bare interface. Due to the large aspect ratio, the $\operatorname{sinc}^{2}$ peak is very tight and shows good agreement with the discretedipole approximation model (see Fig. S4 for curve fitting ${ }^{23}$ ) for a NW length of $6.9 \mu \mathrm{m}$. This agrees well with the values measured using the CCD of $6.5 \pm 0.4 \mu \mathrm{m}$.

The ability of a thin film to increase the directionality of the NW scattering is highlighted in Fig. 3(b), which shows the Fourier space image of a $7.4 \mu \mathrm{m} \mathrm{NW}$ on a glass coverslip, overcoated with $185 \pm 5 \mathrm{~nm}$ of PTFE AF. Again a good agreement with simulations is shown through the line traces and the lobe strength in this instance is 5 times that of the central peak. Thanks to this reduction in low-angle scattering, $43.3 \%$ of the light is scattered within $10^{\circ}$ of the lobe maxima-a $15 \%$ increase from the uncoated NW.

Simulation results revealed in Fig. 4 show that is possible to optimise the fraction of light scattered close to the lobe maxima for a given excitation wavelength by altering
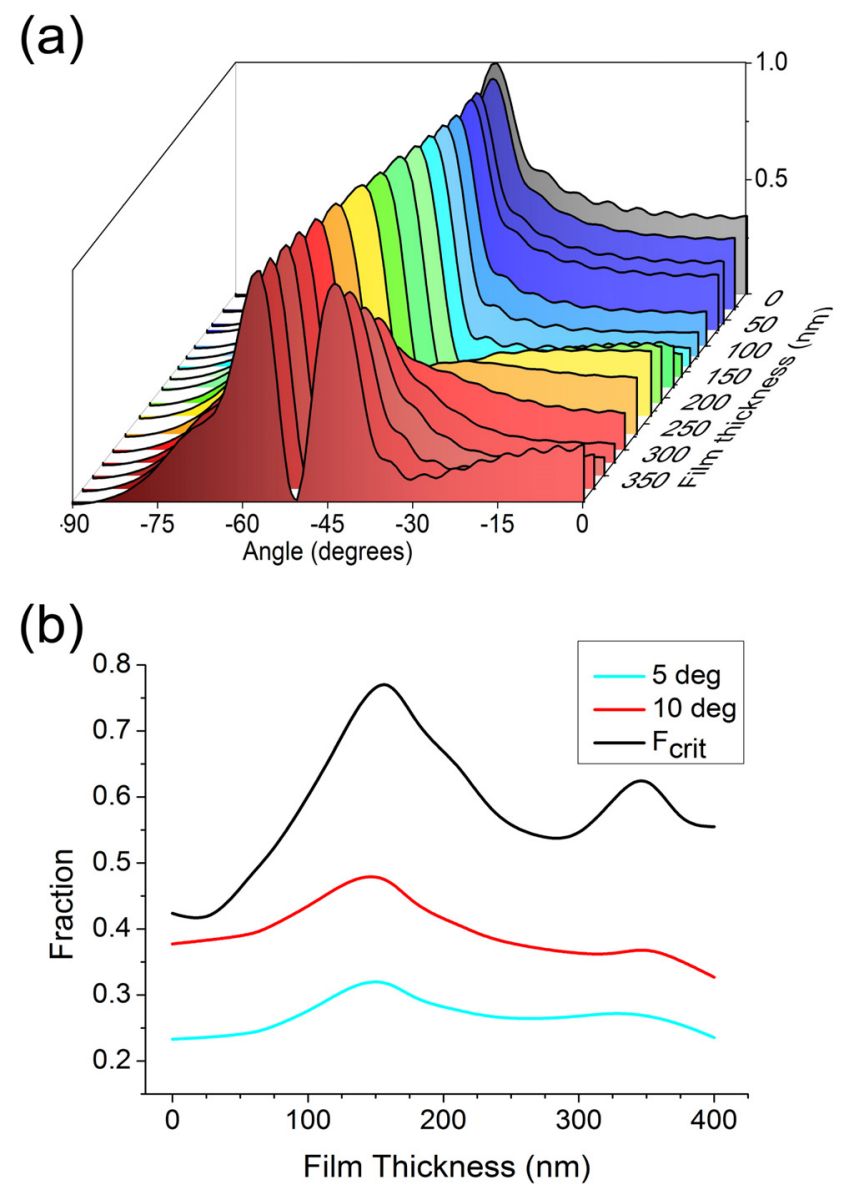

FIG. 4. (a) FDTD simulations showing scattering line traces from $0^{\circ}$ to $90^{\circ}$ for NW's overcoated with various thicknesses of film demonstrating how the scattering pattern develops with $t$. (b) Simulated $t$-dependence of the fraction of $532 \mathrm{~nm}$ light scattered into the substrate within $5^{\circ}$ and $10^{\circ}$ of the lobes as well as the fraction scattered beyond the air-glass critical angle $\left(\mathrm{F}_{\text {crit }}\right)$. 
the film thickness. As $t$ increases, low-angle scattering is reduced until a maximum for angular concentration is reached around $150 \mathrm{~nm}$, beyond which the reemergence of low angle scattering starts to reduce directionality. With thicker films, further lobes appear (as shown in Fig. 4(a), causing additional peaks in Fig. 4(b), but these are all smaller than the original maxima, showing that concentration of scattering around the lobe peaks is maximised for fairly thin films.

It is significant that through the addition of the film the lobe maxima are shifted beyond the air-glass critical angle, which would lead to a large fraction of scattered light being totally internally reflected within the structure. The fraction of light scattered into the substrate beyond the critical angle of $41.8^{\circ},\left(\mathrm{F}_{\text {crit }}\right)$ is shown in Fig. 4(b) (black line). This can be seen to move from $42.5 \%$ for the case of a bare interface to $76.8 \%$ at $t=150 \mathrm{~nm}$, an enhancement of $81 \%$. This could be an important feature for applications such as waveguides and solar cells.

To conclude, we have measured the scattering patterns of NP's and NW's on a glass substrate under various thicknesses of low-index polymer film. The scattering behaviour was altered significantly by the addition of the film above the particles and could be tuned by changing the film thickness. Nanowires were shown to be able to concentrate scattering into a much smaller angular range than NP's. The addition of a film above the NW's produced even stronger directionality which can be enhanced by increasing the NW aspect ratio and optimising the film thickness. The fraction of light scattered past the critical angle of the air-glass interface and hence trapped within the structure can also be maximised by selecting an appropriate film thickness. This demonstrates the potential for fine-tuning the directional scattering of plasmonic nanoparticles for various applications through tailoring the particle shape and dielectric surroundings.
AWP acknowledges support from the UK Engineering and Physical Sciences Research Council.

${ }^{1}$ A. G. Curto, G. Volpe, T. H. Taminiau, M. P. Kreuzer, R. Quidant, and N. F. van Hulst, Science 329, 930 (2010).

${ }^{2}$ N. Hartmann, D. Piatkowski, R. Ciesielski, S. Mackowski, and A. Hartschuh, ACS Nano 7, 10257 (2013).

${ }^{3}$ L. Novotny and N. van Hulst, Nat. Photonics 5, 83 (2011).

${ }^{4}$ T. H. Taminiau, F. D. Stefani, F. B. Segerink, and N. F. van Hulst, Nat. Photonics 2, 234 (2008).

${ }^{5}$ M. Pfeiffer, K. Lindfors, P. Atkinson, A. Rastelli, O. G. Schmidt, H. Giessen, and M. Lippitz, Phys. Status Solidi B 249, 678 (2012).

${ }^{6}$ J. L. Wu, F.-C. Chen, Y.-S. Hsiao, F.-C. Chien, P. Chen, C.-H. Kuo, M. H. Huang, and C.-S. Hsu, ACS Nano 5, 959 (2011).

${ }^{7}$ B. Chen, W. Zhang, X. Zhou, X. Huang, X. Zhao, H. Wang, M. Liu, Y. Lu, and S. Yang, Nano Energy 2, 906 (2013).

${ }^{8}$ P. Spinelli, V. E. Ferry, J. van de Groep, M. van Lare, M. A. Verschuuren,

R. E. I. Schropp, H. A. Atwater, and A. Polman, J. Opt. 14, 024002 (2012).

${ }^{9}$ X. Gu, T. Qiu, W. Zhang, and P. Chu, Nano. Res. Lett. 6, 199 (2011).

${ }^{10}$ M. Garcia, J. Phys. D 44, 283001 (2011).

${ }^{11}$ A. W. Powell, M. B. Wincott, A. A. R. Watt, H. E. Assender, and J. M. Smith, J. Appl. Phys. 113, 184311 (2013).

${ }^{12}$ T. L. Temple and D. Bagnall, J. Appl. Phys. 109, 084343 (2011).

${ }^{13}$ T. L. Temple and D. M. Bagnall, Prog. Photovolt: Res. Appl. 21, 600 (2013).

${ }^{14}$ I. Sersic, C. Tuambilangana, and A. F. Koenderink, New J. Phys. 13, 083019 (2011).

${ }^{15}$ A. G. Curto, T. H. Taminiau, G. Volpe, M. P. Kreuzer, R. Quidant, and N. F. van Hulst, Nat. Commun. 4, 1750 (2013).

${ }^{16}$ V. D. Miljković, T. Shegai, P. Johansson, and M. Käll, Opt. Express 20, 10816 (2012).

${ }^{17}$ A. Drezet, A. Hohenau, D. Koller, A. Stepanov, H. Ditlbacher, B. Steinberger, F. R. Aussenegg, A. Leitner, and J. R. Krenn, Mater. Sci. Eng., B 149, 220 (2008).

${ }^{18}$ A. Drezet and C. Genet, Phys. Rev. Lett. 110, 213901 (2013).

${ }^{19}$ J. D. Jackson, Classical Electrodynamics (Wiley, New York, 1999).

${ }^{20}$ K. Neyts, J. Opt. Soc. Am. A 15, 962 (1998).

${ }^{21}$ K. G. Lee, X. W. Chen, H. Eghlidi, P. Kukura, R. Lettow, A. Renn, and V. S. S. Götzinger, Nat. Photonics 5, 166 (2011).

${ }^{22}$ K. E. Korte, S. E. Skrabalak, and Y. Xia, J. Mater. Chem. 18, 437 (2008).

${ }^{23}$ See supplementary material at http://dx.doi.org/10.1063/1.4866669 for further experimental methodology and curve fitting to scattering plots.

${ }^{24}$ W. Lukosz, J. Opt. Soc. Am. 71, 744 (1981).

${ }^{25}$ T. Shegai, V. D. Miljković, K. Bao, H. Xu, P. Nordlander, P. Johansson, and M. Kall, Nano Lett. 11, 706 (2011). 